\title{
Brane-world Kaluza-Klein reductions and branes on the brane
}

\author{
M. Cvetic \\ Department of Physics and Astronomy, University of Pennsylvania, \\ Philadelphia, Pennsylvania 19104 \\ and SISSA-ISAS INFN, Sezione di Trieste, Via Beirut 2-4, I-34013, Trieste, Italy \\ H. Lü \\ Department of Physics, University of Michigan, Ann Arbor, Michigan 48109 \\ C. N. Pope \\ SISSA-ISAS and INFN, Sezione di Trieste, Via Beirut 2-4, I-34013, Trieste, Italy \\ and Center for Theoretical Physics, Texas A\&M University, \\ College Station, Texas 77843
}

(Received 2 January 2001; accepted for publication 13 February 2001)

We present a systematic study of a new type of consistent "brane-world KaluzaKlein reduction," which describes fully nonlinear deformations of codimension one objects that arise as solutions of a large class of gauged supergravity theories in diverse dimensions, and whose world-volume theories are described by ungauged supergravities with-one half of the original supersymmetry. In addition, we provide oxidations of these ansätz which are in general related to sphere compactified higher dimensional string theory or M-theory. Within each class we also provide explicit solutions of brane configurations localized on the world-brane. We show that at the Cauchy horizon (in the transverse dimension of the consistently KaluzaKlein reduced world-brane) there is a curvature singularity for any configuration with a non-null Riemann curvature or a nonvanishing Ricci scalar that lives in the world-brane. Since the massive Kaluza-Klein modes can be consistently decoupled, they cannot participate in regulating these singularities. (C) 2001 American Institute of Physics. [DOI: 10.1063/1.1377272]

\section{INTRODUCTION}

The conventional way of extracting an effective lower-dimensional theory from a higherdimensional one is by performing a Kaluza-Klein reduction in which the extra dimensions are wrapped up into a compact space such as a torus or a sphere. Provided that the scale size of these internal dimensions is sufficiently small in relation to the energy scale of excitations in the lower dimension, then the mass gap separating the massless modes from the massive ones will be sufficient to ensure that the internal dimensions are essentially unobservable, and the world will appear to be effectively lower dimensional.

If an extra dimension were noncompact then seen from the lower-dimensional viewpoint there would usually be a continuum of modes, with masses extending down to zero. One would normally expect that this would mean that the observable world would be the higher-dimensional one, and that one could not usefully describe it in terms of a lower-dimensional viewpoint. (We cannot usefully view our four-dimensional space-time as being effectively three-dimensional simply by shutting our eyes to the existence of the $z$ axis!) However, it has been shown that under suitable circumstances it may be that the continuous mass eigenvalues for the massive lower-dimensional metric perturbations are distributed in such a way that the effects of the nearly-massless modes is suppressed, implying that the world does in fact appear to be lower-dimensional, with only small modifications to the gravitational forced law appropriate to the lower dimension. ${ }^{1}$ In its original form this Randall-Sundrum II scenario is realised by starting from pure gravity with a negative 
cosmological constant in five dimensions, and patching together two segments of $A d S_{5}$. In horospherical coordinates one has

$$
d \hat{s}_{5}^{2}=e^{-2 k|z|} \eta_{\mu \nu} d x^{\mu} d x^{\nu}+d z^{2},
$$

where the 3-brane is located at $z=0$. (For a review on the global and local space-time structure of the codimension one objects, see Ref. 2.) It was found that gravity is effectively localized on the 3-brane corresponding to the join between the two segments of $A d S_{5} .{ }^{1}$ Specifically, it was shown that the metric fluctuations around the flat Minkowski space-time of the 3-brane are localized near the brane.

More generally, if the flat Minkowski metric on the 3-brane is replaced by any Ricci-flat 4-metric the five-dimensional metric will still, in the bulk, satisfy the Einstein equations with a negative cosmological constant. In other words, one can view

$$
d \hat{s}_{5}^{2}=e^{-2 k|z|} d s_{4}^{2}+d z^{2}
$$

as a Kaluza-Klein reduction ansatz that gives a consistent embedding of four-dimensional pure Einstein gravity in five-dimensional Einstein gravity with a negative cosmological constant. In fact the construction could be extended to give an embedding of four-dimensional $N=1$ ungauged supergravity in five dimensions, by starting from $N=2$ (i.e., minimal) gauged supergravity in $D$ $=5$. Note, however, that the bosonic sector in $D=4$ would still only comprise the metric, and there would be no Maxwell field that could support charged Reissner-Nordström black holes. In particular, it should be noted that one cannot get a Maxwell field as a standard type of KaluzaKlein vector by writing $d \hat{s}_{5}^{2}=e^{-2 k|z|} d s_{4}^{2}+\left(d z+\mathcal{A}_{(1)}\right)^{2}$, since $\partial / \partial z$ is not a Killing vector.

In a recent paper, it was shown that if one instead starts with $N=4$ gauged supergravity in five dimensions, then it is possible to construct a consistent Kaluza-Klein reduction ansatz that gives an embedding of four-dimensional ungauged $N=2$ supergravity on the 3-brane. ${ }^{3}$ This is a new kind of dimensional reduction, which we shall refer to as "brane-world Kaluza-Klein reduction." It should be emphasized that it is nontrivial that a consistent Kaluza-Klein reduction of this sort is possible, (A consistent reduction is one where all the higher-dimensional equations of motion are satisfied provided that the lower-dimensional fields satisfy their equations of motion.) and there is no obvious group-theoretic explanation for why it should work. Two further examples of consistent brane-world Kaluza-Klein reductions were obtained in Ref. 3, describing the embedding of six-dimensional ungauged chiral $N=(1,0)$ supergravity in seven-dimensional $\mathrm{SU}(2)$ gauged $N=2$ supergravity, and the other describing the embedding of five-dimensional ungauged $N=2$ supergravity in six-dimensional $\mathrm{SU}(2)$-gauged $N=2$ supergravity. More generally, it was conjectured that it should be possible to find a consistent brane-world Kaluza-Klein reduction from any gauged supergravity in $D$ dimensions to an ungauged supergravity with half the supersymmetry in $(D-1)$ dimensions. ${ }^{3}$

The purpose of this paper is to provide a systematic construction of consistent brane-world Kaluza-Klein reductions for gauged supergravity theories (with maximal supersymmetry) in diverse dimensions, thus in general leading to the ungauged supergravities with one-half of the original (maximal) gauged supersymmetry. In addition, the studied examples provide compactifications on both AdS and dilatonic codimension one objects. In the first of these examples, in Sec. II, we show that five-dimensional maximal $(N=8) \mathrm{SO}(6)$-gauged supergravity admits a consistent brane-world reduction to four-dimensional ungauged $N=4$ supergravity. Next, in Sec. III, we show that massive type IIA supergravity admits a consistent brane-world reduction to ninedimensional ungauged $N=1$ supergravity. Next, in Sec. IV we show that eight-dimensional maximal $\mathrm{SU}(2)$-gauged supergravity admits a consistent brane-world reduction to seven-dimensional ungauged $N=2$ supergravity. Then, in Sec. V, we show that seven-dimensional maximal SO(5)gauged supergravity admits a consistent brane-world reduction to seven-dimensional ungauged $N=(2,0)$ chiral supergravity.

All the brane-world reductions that were constructed in Ref. 3, and the brane-world reductions of five-dimensional maximal $\mathrm{SO}(6)$-gauged supergravity and seven-dimensional maximal $\mathrm{SO}(5)$ - 
TABLE I. The ungauged supergravities in $(D-1)$ dimensions obtained by brane-world Kaluza-Klein reductions.

\begin{tabular}{rcc}
\hline \hline \multicolumn{1}{c}{$D$} & $D$-dimensional theory & $\begin{array}{c}(D-1) \text {-dimensional theory from } \\
\text { brane-world reduction }\end{array}$ \\
\hline 10 & Massive IIA & $D=9, N=1$ \\
8 & SU(2)-gauged $N=2$ & $D=7, N=2$ \\
7 & SO(5)-gauged $N=4$ & $D=6, N=(2,0)$ \\
6 & SU(2)-gauged $N=2$ & $D=5, N=2$ \\
5 & SO(6) gauged $N=8$ & $D=4, N=4$ \\
\hline \hline
\end{tabular}

gauged supergravity in this paper, are examples where the higher-dimensional theory admits an anti-de Sitter vacuum solution. By contrast, massive type IIA supergravity and the eightdimensional SU(2)-gauged supergravity that we also consider in this paper do not admit anti-de Sitter solutions, but instead they have dilatonic domain walls as their most symmetric "vacuum" solutions. In all the cases, the brane-world Kaluza-Klein reductions can be thought of as fully nonlinear descriptions of deformations around the anti-de Sitter or domain-wall background, in which the $(D-1)$-dimensional Minkowski metric on the $(D-2)$-brane in the $D$-dimensional AdS or domain-wall vacuum is allowed to become arbitrary, along with the other necessary fields that complete the $(D-1)$-dimensional ungauged supergravity multiplet. (We should emphasize that as with any fully nonlinear Kaluza-Klein ansatz, the reduction is not pinned to any specific solution. Although it may sometimes be convenient to think of the AdS or domain-wall solution as playing a preferred role, it is really just one out of an infinity of solutions of the reduced theory.)

A brane-world type of Kaluza-Klein reduction can also be performed in those cases where the $p$-brane cannot trap gravity. A classification of domain walls that can and cannot trap gravity was given in Refs. 4 and 5. In these cases, gravity can arise by placing the $p$-brane on orbifold points ${ }^{6}$ à la Hořava-Witten. ${ }^{7}$

At the level of the supergravity theory, the requirement of the consistency of the brane-world Kaluza-Klein reduction does not discriminate between whether or not the brane is capable of trapping gravity. This is analogous to the situation for a standard Kaluza-Klein reduction on $S^{1}$; at the level of the massless modes, which are the only ones retained in the consistent truncation, one cannot distinguish between an extra dimension that is a circle or an infinite real line. In particular, we shall usually write the brane-world reduction ansatz, as in (2), with an absolutevalue sign for the coordinate $z$, i.e., insisting on a $Z_{2}$ symmetric codimension one ansatz for the transverse dimension. Thus, there is an actual delta function source needed at $z=0$, whose origin lies outside the supergravity Lagrangian description. However, from the mathematical point of view we could perfectly well write the ansatz without the absolute-value sign, thus describing the bulk solution only, which would still correspond to a consistent reduction. In fact now the ansatz will satisfy the equations everywhere, without the need for any external delta-function sources. [However, one would now lose the brane-world interpretation (at $z=0$ ) of the reduced theory.]

We conclude this Introduction with Table I that summarizes the principal results that we obtain in this paper, and those of Ref. 3.

In addition, we shall consider $S^{1}$ reductions of the $D=8$ and $D=7$ gauged supergravities. The former provides a brane-world reduction from $D=7$ that gives the nonchiral $N=(1,1)$ ungauged supergravity in $D=6$, while the latter provides a brane-world reduction from $D=6$ that gives the $N=4$ ungauged theory in $D=5$. The brane-world reduction of the $D=6 \mathrm{SU}(2)$-gauged supergravity was obtained in Ref. 3 , as were the brane-world reductions of the $\mathrm{SU}(2)$-gauged $N=2$ sevendimensional supergravity, and the $\mathrm{SU}(2) \times \mathrm{U}(1)$ gauged $N=4$ five-dimensional supergravity. These two cases are contained within reductions with larger supersymmetries that we consider here. It should be noted that an intrinsic feature of brane-world Kaluza-Klein reductions is that the reduced theory never has more than half the maximal supersymmetry that is allowed in that dimension. This is associated with the fact that there is always a halving of supersymmetry on the brane solution of the higher-dimensional gauged or massive supergravity. 


\section{FOUR-DIMENSIONAL $N=4$ SUPERGRAVITY FROM MAXIMAL FIVE-DIMENSIONAL GAUGED SUPERGRAVITY}

\section{A. Direct reduction from type IIB supergravity}

In Sec. II B, we shall obtain the brane-world embedding of four-dimensional ungauged $N$ $=4$ supergravity in five-dimensional maximal gauged supergravity, thus providing the braneworld Kaluza-Klein compactification in $D=4$ with the maximal allowed ungauged supersymmetry. However, since this five-dimensional theory is rather complicated, we shall begin in the current section by constructing the brane-world embedding of the four-dimensional $N=4$ theory directly in ten-dimensional type IIB supergravity. This exploits the fact that the five-dimensional gauged theory can itself be obtained via an $S^{5}$ reduction from $D=10$. Having done this, we shall then be in a position to re-express our results in terms of a brane-world reduction from $D=5$ to $D=4$. From the five-dimensional viewpoint the fields that we use are the metric, the dilaton and axion [which are singlets under the $\mathrm{SO}(6)$ gauge group], and the two sets of six 2-form potentials. Thus in $D=5$ the 15 Yang-Mills gauge fields and the $10+\overline{10}+20^{\prime}$ of scalars are set to zero.

The bosonic equations of motion of type IIB supergravity can be derived from the Lagrangian,

$$
\begin{aligned}
\mathcal{L}_{10}^{\mathrm{IIB}}= & \hat{R} * \mathbb{1}-\frac{1}{2} \hat{*} d \hat{\phi} \wedge d \hat{\phi}-\frac{1}{2} e^{2 \hat{\phi}_{*}} d \hat{\chi} \wedge d \hat{\chi}-\frac{1}{4} * \hat{F}_{(5)} \wedge \hat{F}_{(5)}-\frac{1}{2} e^{-\hat{\phi}} \hat{F}_{(3)}^{2} \wedge \hat{F}_{(3)}^{2}-\frac{1}{2} e^{\hat{\phi}_{\hat{*}}} \hat{F}_{(3)}^{1} \wedge \hat{F}_{(3)}^{1} \\
& -\frac{1}{2} \hat{A}_{(4)} \wedge d \hat{A}_{(2)}^{1} \wedge d \hat{A}_{(2)}^{2},
\end{aligned}
$$

where $\hat{F}_{(3)}^{2}=d \hat{A}_{(2)}^{2}, \hat{F}_{(3)}^{1}=d \hat{A}_{(2)}^{1}-\hat{\chi} d \hat{A}_{(2)}^{2}, \hat{F}_{(5)}=d \hat{A}_{(4)}-\frac{1}{2} \hat{A}_{(2)}^{1} \wedge d \hat{A}_{(2)}^{2}+\frac{1}{2} \hat{A}_{(2)}^{2} \wedge d \hat{A}_{(2)}^{1}$, and we use carets to denote ten-dimensional fields and the ten-dimensional Hodge dual $\mathscr{*}$. The equations of motion following from the Lagrangian, together with the self-duality condition, are

$$
\begin{aligned}
& \hat{R}_{M N}=\frac{1}{2} \partial_{M} \hat{\phi} \partial_{N} \hat{\phi}+\frac{1}{2} e^{2 \hat{\phi}} \partial_{M} \hat{\chi} \partial_{N} \hat{\chi}+\frac{1}{96} \hat{F}_{M N}^{2}+\frac{1}{4} e^{\hat{\phi}}\left(\left(\hat{F}_{(3)}^{1}\right)_{M N}^{2}\right. \\
& \left.-\frac{1}{12}\left(\hat{F}_{(3)}^{1}\right)^{2} \hat{g}_{M N}\right)+\frac{1}{4} e^{-\hat{\phi}}\left(\left(\hat{F}_{(3)}^{2}\right)_{M N}^{2}-\frac{1}{12}\left(\hat{F}_{(3)}^{2}\right)^{2} \hat{g}_{M N}\right),
\end{aligned}
$$

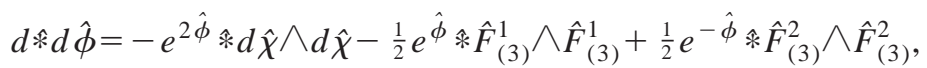

$$
\begin{aligned}
& d\left(e^{2 \hat{\phi}_{*}} d \hat{\chi}\right)=e^{\hat{\phi}_{*}} \hat{F}_{(3)}^{1} \wedge \hat{F}_{(3)}^{2},
\end{aligned}
$$

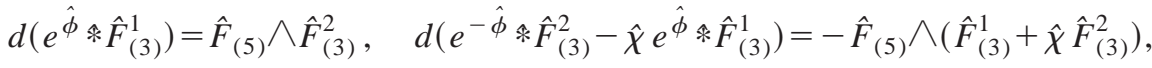

$$
\begin{aligned}
& d\left(* \hat{F}_{(5)}\right)=-\hat{F}_{(3)}^{1} \wedge \hat{F}_{(3)}^{2}, \quad \hat{F}_{(5)}=* \hat{F}_{(5)} .
\end{aligned}
$$

The ungauged four-dimensional $N=4$ supergravity that we are seeking to embed in type IIB supergravity is described by the following Lagrangian (Actually this Lagrangian corresponds to a special truncation of toroidally compactified heterotic string theory where the gauge fields of the original heterotic string are turned off and the momentum and winding modes of the NS-NS sector are identified, thus freezing the internal metric and antisymmetric two-form fields of the six-torus):

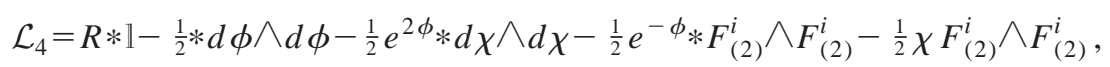

where $1 \leqslant i \leqslant 6$, and $F_{(2)}^{i}=d A_{(1)}^{i}$.

We find that the following is a consistent reduction ansatz that gives the embedding of the four-dimensional $N=4$ theory in type IIB supergravity, 


$$
\begin{gathered}
d \hat{s}_{10}^{2}=e^{-2 k|z|} d s_{4}^{2}+d z^{2}+g^{-2} d \Omega_{5}^{2}, \\
\hat{F}_{(5)}=4 g^{-4} \Omega_{(5)}+4 g e^{-4 k|z|} \epsilon_{(4)} \wedge d z, \\
\hat{A}_{(2)}^{1}=\frac{1}{\sqrt{2}} g^{-1} e^{-k|z|} \mu_{i}\left(e^{-\phi} * F_{(2)}^{i}+\chi F_{(2)}^{i}\right), \\
\hat{A}_{(2)}^{2}=\frac{1}{\sqrt{2}} g^{-1} e^{-k|z|} \mu_{i} F_{(2)}^{i}, \\
\hat{\phi}=\phi, \quad \hat{\chi}=\chi,
\end{gathered}
$$

where $d \Omega_{5}^{2}$ is the metric on the unit 5-sphere, which we can write in terms of six coordinates $\mu_{i}$ that are subject to the constraint $\mu_{i} \mu_{i}=1$, as $d \Omega_{5}^{2}=d \mu_{i} d \mu_{i}$. The 5-form $\Omega_{(5)}$ is the volume form of the metric $d \Omega_{5}^{2}$, and $\epsilon_{(4)}$ is the volume form of the metric $d s_{4}^{2}$. Note that $\Omega_{(5)}$ can be written as

$$
\Omega_{(5)}=\frac{1}{5 !} \epsilon_{i j_{1} \cdots j_{5}} \mu_{i} d \mu_{j_{1}} \wedge \cdots \wedge d \mu_{j_{5}}
$$

The constant $k$ (which we take to be positive) is related to the gauge-coupling constant $g$ of the five-dimensional theory by $k^{2}=g^{2}$. In fact, to be precise, we must have

$$
g=\left\{\begin{array}{cc}
k, & z>0, \\
-k, & z<0 .
\end{array}\right.
$$

Substituting the ansatz (6) into the equations of motion of type IIB supergravity (4), we find that they are all exactly satisfied if and only if the four-dimensional fields $d s_{4}^{2}, \phi, \chi$, and $F_{(2)}^{i}$ satisfy the equations of motion of ungauged $N=4$ supergravity, which can be derived from (5). Note in particular that the six abelian gauge fields $F_{(2)}^{i}$ satisfy the equations of motion,

$$
d\left(e^{-\phi} * F_{(2)}^{i}+\chi F_{(2)}^{i}\right)=0 .
$$

The following results are useful for verifying the consistency of the reduction ansatz. First, we have from (6) that

$$
\begin{gathered}
\hat{F}_{(3)}^{1}=\frac{1}{\sqrt{2}} g^{-1} e^{-k|z|} e^{-\phi} * F_{(2)}^{i} \wedge\left(d \mu_{i}-g \mu_{i} d z\right), \\
\hat{F}_{(3)}^{2}=\frac{1}{\sqrt{2}} g^{-1} e^{-k|z|} F_{(2)}^{i} \wedge\left(d \mu_{i}-g \mu_{i} d z\right) .
\end{gathered}
$$

[Here, we have for convenience of presentation already made use of the fact that the $F_{(2)}^{i}$ satisfy the Bianchi identities $d F_{(2)}^{i}=0$ and the field equations (9); they can, of course, be derived by substituting the ansatz into the ten-dimensional equations of motion.] Next, we can write the ten-dimensional Hodge duals of these field strengths as follows: 


$$
\begin{aligned}
& \hat{*} \hat{F}_{(3)}^{1}=\frac{1}{\sqrt{2}} g^{-5} e^{-k|z|} e^{-\phi} F_{(2)}^{i} \wedge\left(\mu_{i} \Omega_{(5)}-g Z_{i} \wedge d z\right), \\
& \hat{*} \hat{F}_{(3)}^{2}=-\frac{1}{\sqrt{2}} g^{-5} e^{-k|z|} * F_{(2)}^{i} \wedge\left(\mu_{i} \Omega_{(5)}-g Z_{i} \wedge d z\right),
\end{aligned}
$$

where the 4 -form $Z_{i}$ is defined by

$$
Z_{i} \equiv \frac{1}{4 !} \epsilon_{i j k_{1} \cdots{ }_{k 4}} \mu_{j} d \mu_{k_{1}} \wedge \cdots \wedge d \mu_{k_{4}}
$$

This 4-form is the Hodge dual of $d \mu_{i}$ in the unit 5-sphere metric, $*_{5} d \mu_{i}=-Z_{i}$. Note that $Z_{i}$ has the following properties:

$$
d \mu_{i} \wedge Z_{j}=-\left(\delta_{i j}-\mu_{i} \mu_{j}\right) \Omega_{(5)}, \quad d Z_{i}=5 \mu_{i} \Omega_{(5)} .
$$

It is now straightforward to verify that all the type IIB ten-dimensional equations of motion consistently yield the equations of motion of four-dimensional ungauged $N=4$ supergravity; in particular, all the dependence on the coordinates $z$ and $\mu_{i}$ consistently matches in all the equations.

It is worth noting that the $N=4$ gauged supergravity in the four-dimensional world-volume of the D3-brane has an SL(2,Z) electric/magnetic S-duality, with the two scalars $(\phi, \chi)$ parameterizing the $\operatorname{SL}(2, \mathbb{R}) / O(2)$ coset. It is easy to see from the reduction ansatz (6) that this $\operatorname{SL}(2, \mathbb{Z})$ symmetry of the theory in the world-volume of the D3-brane originates from the $\operatorname{SL}(2, Z)$ of the original type IIB theory in $D=10$, which is not an electric/magnetic duality.

It is of interest to see how the brane-world embedding of the four-dimensional $N=4$ supergravity that we have derived here reduces to the $N=2$ supergravity embedding that was constructed in Ref. 3. In the $N=2$ theory there is just one 2-form field strength $F_{(2)}$, and the dilaton $\phi$ and axion $\chi$ are absent. It is easy to see that the equations of motion for $\phi$ and $\chi$ in (5) imply that in order to set $\phi=\chi=0$, we must have

$$
* F_{(2)}^{i} \wedge F_{(2)}^{i}=0, \quad F_{(2)}^{i} \wedge F_{(2)}^{i}=0 .
$$

The minimal nontrivial way to satisfy these conditions is by taking all but two of the six field strengths to vanish, and for the remaining ones, say $F_{(2)}^{1}$ and $F_{(2)}^{2}$, to be related by $F_{(2)}^{2}$ $=* F_{(2)}^{1}$. If we define $F_{(2)}^{2}=* F_{(2)}^{1}=-F_{(2)} / \sqrt{2}$, and at the same time we parameterize the six coordinates $\mu_{i}$ that define the 5 -sphere as

$$
\mu_{1}=\sin \xi \cos \tau, \quad \mu_{2}=-\sin \xi \sin \tau, \quad \mu_{\alpha}=\nu_{\alpha} \cos \xi, \quad(\alpha=3,4,5,6),
$$

where $\nu_{\alpha} \nu_{\alpha}=1$, defining a unit 3-sphere, the ansatz for $d \hat{s}_{10}^{2}, \hat{A}_{(2)}^{1}$, and $\hat{A}_{(2)}^{2}$ in (6) become

$$
\begin{aligned}
& d \hat{s}_{10}^{2}=e^{-2 k|z|} d s_{4}^{2}+d z^{2}+d \xi^{2}+\sin ^{2} \xi d \tau^{2}+\cos ^{2} \xi d \Omega_{3}^{2}, \\
& \hat{A}_{(2)}^{1}+\mathrm{i} \hat{A}_{(2)}^{2}=-\frac{1}{2} g^{-1} e^{-k|z|} \sin \xi e^{-\mathrm{i} \tau}\left(F_{(2)}-\mathrm{i} * F_{(2)}\right) .
\end{aligned}
$$

This is precisely the form of the ansatz found in Ref. 3 for the consistent brane-world embedding of four-dimensional $N=2$ supergravity.

\section{B. Ungauged $D=4, N=4$ from gauged $D=5, N=8$}

In the previous subsection, we considered the reduction from type IIB to the ungauged $N$ $=4$ theory in $D=4$ directly, omitting the intermediate description as a brane-world KK reduction of five-dimensional $N=8$ gauged supergravity on account of the complexity of the five- 
dimensional theory. We can now in fact reinterpret our results as a reduction of the maximal five-dimensional gauged theory. However, in order to avoid the full complexity of this theory, we shall work with a truncation of the full set of five-dimensional fields in which just the metric, the dilaton $\phi$ and axion $\chi$, and the $6+6$ of 2 -form potentials are retained. In other words, we set the 15 Yang-Mills $\mathrm{SO}(6)$ gauge fields and the $10+\overline{10}+20^{\prime}$ of scalars to zero. It should be emphasized that this is in general an inconsistent truncation of the five-dimensional theory. However, we can still work with it provided that we impose the necessary algebraic constraints on the $6+6$ of 2 -form potentials. Of course these constraints are precisely the ones that are satisfied by the brane-world KK reduction ansatz, now expressed simply as a reduction from $D=5$ to $D=4$.

We find that the above truncated five-dimensional theory is obtained from $D=10$ by making the following ansatz for the type IIB fields:

$$
\begin{gathered}
d \hat{s}_{10}^{2}=d s_{5}^{2}+g^{-2} d \Omega_{5}^{2}, \\
\hat{F}_{(5)}=4 g^{-4} \Omega_{(5)}+4 g \epsilon_{(5)}, \\
\hat{A}_{(2)}^{\alpha}=\mu_{i} A_{2}^{i \alpha}, \\
\hat{\phi}=\phi, \quad \hat{\chi}=\chi,
\end{gathered}
$$

where $\alpha=1,2$. We immediately find from the Bianchi identity for $\hat{F}_{(5)}$ that the following equations must be satisfied:

$$
A_{(2)}^{i \alpha} \wedge A_{(2)}^{j \beta} \epsilon_{\alpha \beta}=0, \quad d A_{(2)}^{i \alpha} \wedge A_{(2)}^{j \beta} \epsilon_{\alpha \beta}=0 .
$$

These are the algebraic constraints alluded to above. We must impose them because we have set other fields of the maximal five-dimensional theory to zero, which is in general in conflict with the equations of motion of those fields.

Substituting the ansatz (17) into the remaining equations of motion of type IIB supergravity, and making use of the constraints (18), we find that they consistently imply the following fivedimensional equations:

$$
\begin{gathered}
d A_{(2)}^{i 1}-\chi d A_{(2)}^{i 2}=-g e^{-\phi} * A_{(2)}^{i 2}, \\
d A_{(2)}^{i 2}=g e^{\phi} *\left(A_{(2)}^{i 1}-\chi A_{(2)}^{i 2}\right), \\
d\left(e^{2 \phi} * d \chi\right)=-g^{2} e^{\phi} *\left(A_{(2)}^{i 1}-\chi A_{(2)}^{i 2}\right) \wedge A_{(2)}^{i 2}, \\
d * d \phi=e^{2 \phi} * d \chi \wedge d \chi+\frac{1}{2} g^{2} e^{\phi} *\left(A_{(2)}^{i 1}-\chi A_{(2)}^{i 2}\right) \wedge\left(A_{(2)}^{i 1}-\chi A_{(2)}^{i 2}\right)-\frac{1}{2} g^{2} e^{-\phi} * A_{(2)}^{i 2} \wedge A_{(2)}^{i 2}, \\
R_{\mu \nu}=\frac{1}{2} \partial_{\mu} \phi \partial_{\nu} \phi+\frac{1}{2} e^{2 \phi} \partial_{\mu} \chi \partial_{\nu} \chi+\frac{1}{2} g^{2}\left[e^{\phi}\left(A_{\mu \rho}^{i 1}-\chi A_{\mu \rho}^{i 2}\right)\left(A_{\nu}^{i 1 \rho}-\chi A_{\nu}^{i 2 \rho}\right)+e^{-\phi} A_{\mu \rho}^{i 2} A_{\nu}^{i 2 \rho}\right] .
\end{gathered}
$$

$\left[A_{(2)}^{i 1}\right.$ and $A_{(2)}^{i 2}$ denote $A_{(2)}^{i \alpha}$ with $\alpha=1$ and $\alpha=2$, respectively.] These equations, together with the constraints (18), are precisely equivalent to those of maximal five-dimensional gauged supergravity, after setting the Yang-Mills fields and the $10+\overline{10}+20^{\prime}$ of scalars to zero. The $6+6$ of 2 -form fields $A_{(2)}^{i \alpha}$ satisfy first-order equations of motion, known as "odd-dimensional self-duality equations.' These, together with the constraint equations (18), imply that the trace of the 2-form contributions in the energy-momentum tensor vanishes; $e^{\phi}\left(A_{\mu \nu}^{i 1}-\chi A_{\mu \nu}^{i 2}\right)\left(A^{i 1 \mu \nu}-\chi A^{i 2 \mu \nu}\right)$ $+e^{-\phi} A_{\mu \nu}^{i 2} A^{i 2 \mu \nu}=0$. Note that the imposition of the constraints (18) is sufficient to ensure that all the type IIB equations of motion are consistently satisfied by the ansatz (17), including the internal and mixed components of the Einstein equations.

It is useful to observe that the equations of motion (19) can be derived from the Lagrangian, 


$$
\begin{aligned}
\mathcal{L}_{5}= & R * 1-\frac{1}{2} * d \phi \wedge d \phi-\frac{1}{2} e^{2 \phi} * d \chi \wedge d \chi-\frac{1}{2} g^{2} e^{\phi} *\left(A_{(2)}^{i 1}-\chi A_{(2)}^{i 2}\right) \wedge\left(A_{(2)}^{i 1}-\chi A_{(2)}^{i 2}\right) \\
& -\frac{1}{2} g^{2} e^{-\phi} * A_{(2)}^{i 2} \wedge A_{(2)}^{i 2}-g d A_{(2)}^{i 1} \wedge A_{(2)}^{i 2}+12 g^{2} * 1 .
\end{aligned}
$$

Finally, we note that the brane-world Kaluza-Klein reduction of the previous subsection, now expressed as a reduction from $D=5$ to $D=4$, is given by

$$
\begin{gathered}
d s_{5}^{2}=e^{-2 k|z|} d s_{4}^{2}+d z^{2}, \\
A_{(2)}^{i 1}=\frac{1}{\sqrt{2}} g^{-1} e^{-k|z|}\left(e^{-\phi} * F_{(2)}^{i}+\chi F_{(2)}^{i}\right), \\
A_{(2)}^{i 2}=\frac{1}{\sqrt{2}} g^{-1} e^{-k|z|} F_{(2)}^{i},
\end{gathered}
$$

with $\phi$ and $\chi$ just reducing directly. One can easily verify that this reduction ansatz is indeed compatible with the constraints (18).

\section{Branes on the D3-brane}

One can construct electric and magnetic black holes, strings and instantons in $D=4, N=4$ supergravity. They become branes on the D3-brane (in the near-horizon region) when they are lifted back to $D=10$. We analyze these solutions in this section.

Case 1: SL(2,Z) dyonic black holes on the D3-brane: We can use one of the six 2-form field strengths to construct an electric or magnetic black hole. As a concrete example, let us consider an electric black hole supported by the field strength $F_{(2)}^{1}$. Once the solution is lifted back to $D$ $=10$, it becomes

$$
\begin{gathered}
d \hat{s}_{10}^{2}=e^{-2 k|z|}\left[-H^{-1} d t^{2}+H\left(d r^{2}+r^{2} d \Omega_{2}^{2}\right)\right]+d z^{2}+g^{-2} d \Omega_{5}^{2}, \\
\hat{F}_{(5)}=4 g^{-4} \Omega_{5}+4 g e^{-4 k|z|} r^{2} H d t \wedge d r \wedge \Omega_{2}, \\
\hat{A}_{(2)}^{1}=\frac{Q}{\sqrt{2}} g^{-1} e^{-k|z|} \mu_{1} \Omega_{2}, \\
\hat{A}_{(2)}^{2}=\frac{1}{\sqrt{2}} g^{-1} e^{-k|z|} \mu_{1} d t \wedge d H^{-1} \\
e^{\phi}=H, \quad H=1+\frac{Q}{r}
\end{gathered}
$$

where $\mu_{1}$ is one of the coordinates $\mu_{i}$ for $S^{5}$ appearing in the ansatz (17), corresponding to our choice to consider a black hole supported by $F_{(2)}^{1}$. Starting with the electric black hole, we can then apply the $\operatorname{SL}(2, R)$ symmetry to get a multiplet of dyonic black holes, where the electric and magnetic charges are carried by the same 2-form field strength. The metric of this dyonic solution remains unchanged, but the charge configuration alters.

Case 2: Threshold dyonic black holes on the D3-brane: In $D=4, N=4$ supergravity, one can also construct a multicharge black hole solution, where the electric charge is carried by one 2-form field strength, say $F_{(2)}^{1}$, and the magnetic charge is carried by another, say $F_{(2)}^{2} \cdot{ }^{8}$ [Note that the generating technique, as employed, for example, for the four-charge solution ${ }^{9}$ of toroidally compactified heterotic string, may allow for a construction of more general dyonic black holes with all the $\mathrm{U}(1)$ charges turned on.] Lifting this solution back to $D=10$, it becomes 


$$
\begin{gathered}
d \hat{s}_{10}^{2}=e^{-2 k|z|}\left[-\left(H_{1} H_{2}\right)^{-1} d t^{2}+H_{1} H_{2}\left(d r^{2}+r^{2} d \Omega_{2}^{2}\right)\right]+d z^{2}+g^{-2} d \Omega_{5}^{2}, \\
\hat{F}_{(5)}=4 g^{-4} \Omega_{5}+4 g e^{-4 k|z|} r^{2}\left(H_{1} H_{2}\right) d t \wedge d r \wedge d \Omega_{2}, \\
\hat{A}_{(2)}^{1}=\frac{1}{\sqrt{2}} g^{-1} e^{-k|z|}\left(Q_{e} \mu_{1} \Omega_{2}+\mu_{2} d t \wedge d H_{2}^{-1}\right), \\
\hat{A}_{(2)}^{2}=\frac{1}{\sqrt{2}} g^{-1} e^{-k|z|}\left(\mu_{1} d t \wedge d H_{1}^{-1}+\mu_{2} Q_{m} \Omega_{2}\right), \\
e^{\phi}=\frac{H_{1}}{H_{2}}, \quad H_{1}=1+\frac{Q_{e}}{r}, \quad H_{2}=1+\frac{Q_{m}}{r} .
\end{gathered}
$$

Case 3: String on the D3-brane: A magnetic string (four-dimensional domain wall), supported by the axion, exists in the four-dimensional supergravity theory. Lifting this solution back to $D$ $=10$, we have a string living on the D3-brane,

$$
\begin{gathered}
d \hat{s}_{10}^{2}=e^{-2 k|z|}\left[-d t^{2}+d x^{2}+H\left(d r^{2}+r^{2} d \theta^{2}\right)\right]+d z^{2}+g^{-2} d \Omega_{5}^{2}, \\
\hat{F}_{(5)}=4 g^{-4} \Omega_{(5)}+4 g e^{-4 k|z|} r H d t \wedge d x \wedge d r \wedge d \theta, \\
e^{\hat{\phi}}=H^{-1}, \quad \chi=Q \theta, \\
H=1+Q \log r .
\end{gathered}
$$

This solution is a non-standard intersection of a D3-brane and D7-brane, where there is no overall transverse space. It should be distinguished from the solution describing a D3-brane in the D7brane, which has a two-dimensional overall transverse space.

Case 4: Instanton on the D3-brane: The axion in the $D=4$ theory also supports a BPS instanton solution when the theory is Euclideanized. The axion $\chi$ becomes imaginary under this procedure, the metric $d s_{4}^{2}$ becomes purely flat Euclidean space, and $\hat{F}_{(5)}$ becomes complex, since in ten Euclidean dimensions a real 5-form cannot be self-dual.

\section{III. $N=1$ SUPERGRAVITY IN $D=9$ FROM MASSIVE TYPE IIA}

\section{A. D8-brane in massive type IIA theory}

The highest dimensional D-brane that can be found in any supergravity theory is the D8-brane in massive type IIA supergravity. This theory was constructed in Ref. 10, but in a formulation where there is not a straightforward massless limit to ordinary type IIA supergravity. However, it is simply a matter of performing a field redefinition to resolve this problem. ${ }^{11}$ The Lagrangian for the bosonic sector of the massive type IIA supergravity can then be written as the following differential form: ${ }^{12}$

$$
\begin{aligned}
& \mathcal{L}_{10}=\hat{R} \hat{*} 1-\frac{1}{2} * d \hat{\phi} \wedge d \hat{\phi}-\frac{1}{2} e^{3 / 2 \hat{\phi}_{*}} \hat{F}_{(2)} \wedge \hat{F}_{(2)}-\frac{1}{2} e^{-\hat{\phi}_{*}} \hat{F}_{(3)} \wedge \hat{F}_{(3)}-\frac{1}{2} e^{1 / 2 \hat{\phi}_{*}} \hat{F}_{(4)} \wedge \hat{F}_{(4)} \\
& -\frac{1}{2} d \hat{A}_{(3)} \wedge d \hat{A}_{(3)} \wedge \hat{A}_{(2)}-\frac{1}{6} m d \hat{A}_{(3)} \wedge\left(\hat{A}_{(2)}\right)^{3}-\frac{1}{40} m^{2}\left(\hat{A}_{(2)}\right)^{5}-\frac{1}{2} m^{2} e^{5 / 2 \hat{\phi}_{*}} \mathbb{1},
\end{aligned}
$$

where the field strengths are given in terms of potentials by

$$
\begin{gathered}
\hat{F}_{(2)}=d \hat{A}_{(1)}+m \hat{A}_{(2)}, \quad \hat{F}_{(3)}=d \hat{A}_{(2)}, \\
\hat{F}_{(4)}=d \hat{A}_{(3)}+\hat{A}_{(1)} \wedge d \hat{A}_{(2)}+\frac{1}{2} m \hat{A}_{(2)} \wedge \hat{A}_{(2)} .
\end{gathered}
$$


The Bianchi identities for the field strengths are therefore

$$
d \hat{F}_{(2)}=m \hat{F}_{(3)}, \quad d \hat{F}_{(3)}=0, \quad d \hat{F}_{(4)}=\hat{F}_{(2)} \wedge \hat{F}_{(3)},
$$

and the field equations are

$$
\begin{gathered}
d \hat{F}_{(6)}=-\hat{F}_{(3)} \wedge \hat{F}_{(4)}, \quad d \hat{F}_{(8)}=-\hat{F}_{(3)} \wedge \hat{F}_{(6)}, \\
d \hat{F}_{(7)}=-\frac{1}{2} \hat{F}_{(4)} \wedge \hat{F}_{(4)}-m \hat{F}_{(8)}-\hat{F}_{(2)} \wedge \hat{F}_{(6)}, \\
d * d \hat{\phi}=-\frac{1}{4} \hat{F}_{(4)} \wedge \hat{F}_{(6)}-\frac{3}{4} \hat{F}_{(2)} \wedge \hat{F}_{(8)}-\frac{1}{2} \hat{F}_{(3)} \wedge \hat{F}_{(7)}+\frac{5}{4} m^{2} e^{5 / 2 \hat{\phi}} \hat{*},
\end{gathered}
$$

where we have defined the dual field strengths

$$
\hat{F}_{(6)} \equiv e^{1 / 2 \hat{\phi}_{*}} \hat{F}_{(4)}, \quad \hat{F}_{(8)} \equiv e^{3 / 2 \hat{\phi}_{*}} \hat{F}_{(2)}, \quad \hat{F}_{(7)} \equiv e^{-\hat{\phi}_{*}} \hat{F}_{(3)} .
$$

This massive type IIA theory supports a "vacuum" solution, namely, the D8-brane,

$$
d \hat{s}_{10}^{2}=W^{2 / 25} d x^{\mu} d x_{\mu}+d z^{2}, \quad e^{\hat{\phi}}=W^{-(4 / 5)},
$$

where the one-dimensional harmonic function is given by

$$
W=1+k|z|, \quad k^{2}=\frac{625}{256} m^{2}
$$

In fact the sign of $m$ must be opposite on opposite sides of the domain wall,

$$
g=\left\{\begin{array}{l}
\frac{16}{25} k, \quad z>0, \\
-\frac{16}{25} k, \quad z<0,
\end{array}\right.
$$

where $k$ is assumed to be positive. This means that one cannot strictly speaking view the domain wall as a solution within the massive type IIA theory as formulated in Ref. 10, since there $m$ is a fixed parameter in the Lagrangian. However, the theory can be re-expressed in a formulation where $m$ is replaced by a 10 -form field, with the mass parameter now arising as a constant of integration. It now makes sense for the parameter to be only piecewise constant. In what follows, we shall implicitly assume that we are working with this reformulation of the theory, which allows (32) to hold.

Note that the nine-dimensional flat Minkowskian spacetime $d x^{\mu} d x_{\mu}$ of the solution (30) can be replaced by any Ricci-flat Minkowski-signatured space-time. ${ }^{13}$ On the other hand, it was observed that domain walls associated with $\mathrm{D} p$-branes with $p \geqslant 6$ cannot trap gravity. Nevertheless, one can still obtain gravity on the world-volume in such a case by locating the branes at orbifold points, so that the space-time is compact. ${ }^{6}$ In this case, we would expect that the resulting theory on the world-volume of the D8-brane would be the ungauged $N=1, D=9$ supergravity. We shall prove in the next subsection that this can indeed be obtained from the massive type IIA theory via a consistent brane-world Kaluza-Klein reduction.

\section{B. $N=1$ supergravity in $D=9$ from massive type IIA}

We find that the following Kaluza-Klein ansatz for the ten-dimensional massive type IIA fields yields a consistent reduction to nine dimensions, 


$$
\begin{gathered}
d \hat{s}_{10}^{2}=e^{-(5 / 16) \sqrt{(2 / 7)} \phi} W^{(2 / 25)} d s_{9}^{2}+e^{(35 / 16) \sqrt{(2 / 7)} \phi} d z^{2}, \\
\hat{A}_{(1)}=0, \quad \hat{A}_{(2)}=\frac{1}{2 m} W^{16 / 25} F_{(2)}, \quad \hat{A}_{(3)}=\frac{1}{4 m} W^{32 / 25} F_{(3)}, \\
e^{\hat{\phi}}=W^{-(4 / 5)} e^{-(7 / 8) \sqrt{(2 / 7)} \phi},
\end{gathered}
$$

where $W$ is given by (31) and $g$ is related to $k$ by (32). Substituting this ansatz into the massive type IIA equations of motion, we find that they are all satisfied provided that the nine-dimensional fields $d s_{9}^{2}, \varphi, F_{(2)}=d A_{(1)}$ and $F_{(3)}=d A_{(2)}-\frac{1}{2} A_{(1)} \wedge F_{(2)}$ satisfy the equations of motion of ninedimensional ungauged simple supergravity. These equations can be derived from the Lagrangian,

$$
\mathcal{L}_{9}=R * 1-\frac{1}{2} * d \phi \wedge d \phi-\frac{1}{2} e^{-\sqrt{(8 / 7)} \phi} * F_{(3)} \wedge F_{(3)}-\frac{1}{2} e^{-\sqrt{(2 / 7)} \phi} * F_{(2)} \wedge F_{(2)}
$$

\section{Branes on the D8-brane}

Having consistently embedded the ungauged $N=1, D=9$ supergravity in massive type IIA supergravity, we can lift all the solutions of this nine-dimensional theory back to $D=10$. The nine-dimensional theory supports BPS $p$-branes such as the string, 4-brane, black hole and 5-brane. These solutions are straightforward and well known. When they are lifted back to massive type IIA supergravity using the ansatz (33), they can be viewed as branes living on the D8-brane.

Case 1: String on the D8-brane: The solution of the $D=9$ string lifted back to $D=10$ becomes

$$
\begin{gathered}
d \hat{s}_{10}^{2}=W^{(2 / 25)}\left[H^{-(5 / 8)}\left(-d t^{2}+d x^{2}\right)+H^{3 / 8}\left(d r^{2}+r^{2} d \Omega_{6}^{2}\right)\right]+H^{-(5 / 8)} d z^{2}, \\
\hat{A}_{(3)}=\frac{1}{4 m} W^{32 / 25} d t \wedge d x \wedge d H^{-1}, \\
e^{\hat{\phi}}=W^{-(4 / 5)} H^{1 / 4}, \quad H=1+\frac{Q}{r^{5}} .
\end{gathered}
$$

This solution can be viewed as a D2-brane ending on the D8-brane, with the end points forming a string. To see this, it is helpful to introduce a new coordinate $y$ in place of $z$, defined by $d y$ $=\frac{24}{25} W^{-(1 / 25)} d z$, and hence

$$
W=(1+k|y|)^{25 / 24} \text {. }
$$

Using this variable, the $y$-dependence of the metric is extracted as an overall conformal factor, and we have

$$
d \hat{s}_{10}^{2}=(1+k|y|)^{1 / 12}\left[H^{-(5 / 8)}\left(-d t^{2}+d x^{2}+d y^{2}\right)+H^{3 / 8}\left(d r^{2}+r^{2} d \Omega_{6}^{2}\right)\right] .
$$

Case 2: 4-brane on the D8-brane: The $D=9$ 4-brane solution (the magnetic dual of the string solution), lifted back to $D=10$, becomes

$$
\begin{aligned}
& d \hat{s}_{10}^{2}=W^{2 / 25}\left[H^{-(3 / 8)} d x^{\mu} d x_{\mu}+H^{5 / 8}\left(d r^{2}+r^{2} d \Omega_{3}^{2}\right)\right]+H^{5 / 8} d z^{2}, \\
& \hat{A}_{(3)}=\frac{Q}{4 m} W^{32 / 25} \Omega_{3}, \\
& e^{\hat{\phi}}=W^{-(4 / 5)} H^{-(1 / 4)}, \quad H=1+\frac{Q}{r^{2}} .
\end{aligned}
$$

Using the same coordinate transformation (36), the metric can be re-expressed as 


$$
d \hat{s}_{10}^{2}=(1+k|y|)^{1 / 12}\left[H^{-(3 / 8)} d x^{\mu} d x_{\mu}+H^{5 / 8}\left(d y^{2}+d r^{2}+r^{2} d \Omega_{3}^{2}\right)\right] .
$$

Thus the solution can be viewed as a D4-brane intersecting with a D8-brane, with the D4-brane uniformly delocalized on the one-dimensional transverse space of the D8-brane.

In Case 1 above, the intersection of the D2-brane and the D8-brane is such that the overall world-volume is a string, and the solution describes a D2-brane ending on the D8-brane. In Case 2 , the intersection of the D4-brane and D8-brane is such that the overall world-volume is the entire 4-brane, and so the solution describes a D4-brane living in the D8-brane.

Case 3: Black hole on the D8-brane: The black hole solution of the $D=9$ theory can be lifted to $D=10$, where it becomes

$$
\begin{gathered}
d \hat{s}_{10}^{2}=W^{(2 / 25)}\left[-H^{-(13 / 8)} d t^{2}+H^{3 / 8}\left(d r^{2}+r^{2} d \Omega_{7}^{2}\right)\right]+H^{-(5 / 8)} d z^{2}, \\
\hat{A}_{(2)}=\frac{1}{2 m} W^{(16 / 25)} d t \wedge d H^{-1}, \\
e^{\hat{\phi}}=W^{-(4 / 5)} H^{1 / 4}, \quad H=1+\frac{Q}{r^{6}} .
\end{gathered}
$$

Using (36), the metric can be cast into the form,

$$
d \hat{s}_{10}^{2}=(1+k|y|)^{1 / 12}\left[-H^{-(13 / 8)} d t^{2}+H^{-(5 / 8)} d y^{2}+H^{3 / 8}\left(d r^{2}+r^{2} d \Omega_{7}^{2}\right)\right] .
$$

The solution can be viewed as the intersection of an NS-NS string and a D0-brane with the D8-brane. In particular, the string NS-NS string ends on the D8-brane whilst the D0-brane lives in the D8-brane. To see this, we note that a standard solution for the intersection of a string and a D0-brane would be

$$
d s_{10}^{2}=-H_{0}^{-(7 / 8)} H_{1}^{-(3 / 4)} d t^{2}+H_{0}^{1 / 8} H_{1}^{-(3 / 4)} d u^{2}+H_{0}^{1 / 8} H_{1}^{1 / 4} d \mathbf{y}^{2},
$$

where $H_{0}$ and $H_{1}$ are independent harmonic functions on the eight-dimensional common transverse space of the $\mathbf{y}$ coordinates. If these two harmonic functions are set equal, $H_{0}=H_{1}=H$, then we obtain the structure found in (41).

Case 4: 5-brane on the D8-brane: The 5-brane solution (the magnetic dual of the black hole) can be lifted to $D=10$, where it becomes

$$
\begin{gathered}
d \hat{s}_{10}^{2}=W^{2 / 25}\left[H^{-(3 / 8)} d x^{\mu} d x_{\mu}+H^{13 / 8}\left(d r^{2}+r^{2} d \Omega_{2}\right)\right]+H^{5 / 8} d z^{2}, \\
\hat{A}_{(2)}=\frac{Q}{2 m} W^{16 / 25} \Omega_{2}, \\
e^{\hat{\phi}=W^{-(4 / 5)}} H^{-(1 / 4)}, \quad H=1+\frac{Q}{r} .
\end{gathered}
$$

Using the redefinition (36), the metric can be cast into the form

$$
d \hat{s}_{10}^{2}=(1+k|y|)^{1 / 12}\left(-H^{-(3 / 8)} d x^{\mu} d x_{\mu}+H^{5 / 8} d y^{2}+H^{13 / 8}\left(d r^{2}+r^{2} d \Omega_{2}^{2}\right)\right) .
$$

The solution can be viewed as an intersection of a NS-NS 5-brane and a D6-brane with the D8-brane. In particular, the NS-NS 5-brane lives in the D8-brane, whilst the D6-brane ends on the D8-brane. (The standard solution for the intersection of a 5-brane and a 6-brane would be of the form,

$$
d s_{10}^{2}=H_{5}^{-(1 / 4)} H_{6}^{-(1 / 8)} d x^{\mu} d x_{\mu}+H_{5}^{3 / 4} H_{6}^{-(1 / 8)} d u^{2}+H_{5}^{3 / 4} H_{6}^{7 / 8} d \mathbf{y}^{3},
$$


where $H_{5}$ and $H_{6}$ are independent harmonic functions on the common transverse 3-space of the $\mathbf{y}$ coordinates. In our case, the two harmonic functions are equal, $H_{5}=H_{6}=H$.)

Note that it is straightforward also to construct pp-wave and Taub-NUT solutions on the world-volume of the D8-brane.

\section{REDUCTIONS OF SU(2)-GAUGED $D=8$ SUPERGRAVITY}

\section{A. Brane-world reduction to $D=7$}

Although there is no gauged supergravity in eight dimensions that admits a maximallysymmetric $\mathrm{AdS}_{8}$ solution, there is a gauged theory that arises from the dimensional reduction of eleven-dimensional supergravity on $S^{3} .{ }^{14}$ Since only the gauge bosons of the left-acting SU(2) of the $\mathrm{SO}(4) \sim \mathrm{SU}(2) \times \mathrm{SU}(2)$ are retained in the truncation, the consistency of this reduction to $D=8$ is guaranteed by the standard group-theoretic arguments of Ref. 15 . The theory can also be obtained from the $S^{2}$ reduction of type IIA theory, and the $\mathrm{SU}(2)$ is the isometry group of the 2 -sphere. The eleventh coordinate is the fibre coordinate of the $S^{3}$, which can be viewed as a U(1) bundle over $S^{2}{ }^{16}$ The eight-dimensional theory admits a dilatonic 6-brane domain-wall solution, and this provides a starting-point for the construction of a brane-world Kaluza-Klein reduction to $D=7$.

The bosonic sector of the eight-dimensional theory contains the metric, a dilatonic scalar $\varphi$, five further scalars that can be parameterized by a unimodular $3 \times 3$ symmetric matrix $T_{i j}$, the $\mathrm{SU}(2)$ Yang-Mills potentials $A_{(1)}^{i}$, three 2-form potentials $B_{(2)}^{i}$, and a 3 -form potential $A_{(3)}$. The description of the theory is a little involved, but the majority of the complications come from the scalars $T_{i j}$ and the Yang-Mills potentials $A_{(1)}^{i}$ that will in fact be set to zero in our brane-world Kaluza-Klein reduction to $D=7$. It is not in general consistent in $D=8$ to set $T_{i j}=\delta_{i j}$ and $A_{(1)}^{i}=0$ while keeping all the other fields nonvanishing, since the retained fields will act as sources for those that are set to zero. However, since in our brane-world reduction to $D=7$ the ansatz for the remaining nonvanishing eight-dimensional fields will be such that these source terms vanish, it is sufficient for our purposes to present the truncated eight-dimensional theory, together with constraints that will be identically satisfied by the brane-world reduction ansatz. These constraints are precisely the conditions that the sources that would have excited the truncated fields should be zero.

It is in fact easy to obtain this truncation of the eight-dimensional gauged theory as an $S^{3}$ reduction from $D=11$. The ansatz is given by

$$
\begin{gathered}
d \hat{s}_{11}^{2}=e^{-(1 / 3) \varphi} d s_{8}^{2}+e^{2 / 3 \varphi} g^{-2} d \Omega_{3}^{2}, \\
\hat{A}_{(3)}=A_{3}+\frac{1}{2} g^{-1} B_{(2)}^{i} \wedge \sigma_{i} .
\end{gathered}
$$

The quantities $\sigma_{i}$ are the three left-invariant 1-forms on the group manifold $\mathrm{SU}(2)$, satisfying $d \sigma_{i}=-\frac{1}{2} \epsilon_{i j k} \sigma_{j} \wedge \sigma_{k}$. In terms of these, the unit metric on $S^{3}$ can be written as $d \Omega_{3}^{2}=\frac{1}{4} \sigma_{i} \sigma_{i}$. Substituting the ansatz into the bosonic equations of motion of eleven-dimensional supergravity,

$$
d \hat{F}_{(4)}=\frac{1}{2} \hat{F}_{(4)} \wedge \hat{F}_{(4)}, \quad \hat{R}_{M N}=\frac{1}{12}\left(\hat{F}_{M N}^{2}-\frac{1}{12} \hat{F}_{(4)}^{2} \hat{g}_{M N}\right),
$$

we find that the field equation for $\hat{F}_{(4)}$ implies

$$
\begin{gathered}
d\left(e^{\varphi} * F_{(4)}\right)=-2 g B_{(2)}^{i} \wedge G_{(3)}^{i}, \\
d * G_{(3)}^{i}=-4 g^{2} e^{-\varphi_{*}} B_{(2)}^{i}-2 g F_{(4)} \wedge B_{(2)}^{i}-g \epsilon_{i j k} G_{(3)}^{j} \wedge G_{(3)}^{g}, \\
F_{(4)} \wedge F_{(4)}=0,
\end{gathered}
$$


where $F_{(4)} \equiv d A_{(3)}$ and $G_{(3)}^{i} \equiv d B_{(2)}^{i}$. Note that the last equation in (48) is one of the constraints that results from our having truncated out the $T_{i j}$ and $A_{(1)}^{i}$ fields. From the Einstein equation in (47), we obtain the following eight-dimensional equations of motion:

$$
\begin{gathered}
R_{\mu \nu}=\frac{1}{2} \partial_{\mu} \varphi \partial_{\nu} \varphi-\frac{1}{6} \square \varphi g_{\mu \nu}+\frac{1}{12} e^{\varphi}\left[F_{\mu \rho \sigma \lambda} F_{\nu}^{\rho \sigma \lambda}-\frac{1}{12} F_{(4)}^{2} g_{\mu \nu}\right] \\
+\frac{1}{4}\left[G_{\mu \rho \sigma}^{i} G_{\nu}^{i \rho \sigma}-\frac{1}{9}\left(G_{(3)}^{i}\right)^{2} g_{\mu \nu}\right]+2 g^{2} e^{-\varphi}\left[B_{\mu \rho}^{i} B_{\nu}^{i \rho}-\frac{1}{6}\left(B_{(2)}^{i}\right)^{2} g_{\mu \nu}\right], \\
\square \varphi=6 g^{2} e^{-\varphi}+\frac{1}{48} e^{\varphi} F_{(4)}^{2}-g^{2} e^{-\varphi}\left(B_{(2)}^{i}\right)^{2},
\end{gathered}
$$

together with the further constraints

$$
\begin{gathered}
e^{\varphi} F_{\mu \nu \rho \sigma} G^{i \nu \rho \sigma}+6 g \epsilon_{i j k} G_{\mu \rho \sigma}^{j} B^{k \rho \sigma}=0, \\
e^{\varphi} G_{\mu \nu \rho}^{i} G^{j \mu \nu \rho}-12 g^{2} B_{\mu \nu}^{i} B^{j \mu \nu}=0 .
\end{gathered}
$$

These come from the mixed and the purely internal components of the eleven-dimensional Einstein equation, respectively.

The eight-dimensional equations of motion admit a domain-wall "ground-state" solution, where all fields except $d s_{8}^{2}$ and $\varphi$ are set to zero, and

$$
d s_{8}^{2}=W^{2 / 3} d x \cdot d x+d z^{2}, \quad e^{\varphi}=W^{2},
$$

where

$$
W=1+k|z|, \quad k^{2}=\frac{9}{4} g^{2} .
$$

Specifically,

$$
g=\left\{\begin{array}{l}
\frac{2}{3} k, \quad z>0, \\
-\frac{2}{3} k, \quad z<0 .
\end{array}\right.
$$

(As usual, $g$ is allowed to have the necessary sign-change across the domain-wall provided that one thinks of obtaining the eight-dimensional gauged theory as an $S^{3}$ reduction from $D=11$, since then $g$ arises as a constant of integration, rather than as a fixed parameter in the eight-dimensional Lagrangian.)

This motivates the construction of the following brane-world reduction ansatz, to give ungauged seven-dimensional $N=2$ supergravity from the gauged eight-dimensional theory,

$$
\begin{gathered}
d \hat{s}_{8}^{2}=e^{-(1 / 2 \sqrt{5}) \phi} W^{2 / 3} d s_{7}^{2}+e^{(\sqrt{5} / 2) \phi} d z^{2}, \\
\hat{B}_{(2)}^{i}=\frac{1}{2 \sqrt{2}} g^{-1} W^{4 / 3} F_{(2)}^{i}, \\
\hat{A}_{(3)}=A_{(3)}, \quad e^{\hat{\varphi}}=W^{2} e^{(\sqrt{5} / 2) \phi},
\end{gathered}
$$

where we have now placed hats on all the eight-dimensional fields.

Substituting this ansatz into the equations of motion for the eight-dimensional gauged theory given above, we find that they are satisfied provided that the seven-dimensional fields satisfy the equations of motion of ungauged seven-dimensional $N=2$ supergravity. Specifically, these can be derived from the Lagrangian, 


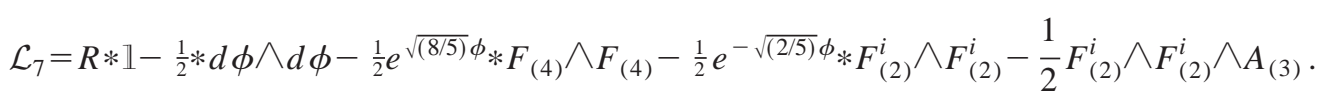

It should be noted also that the ansatz (54) identically satisfies the constraint equations in (48) and (50), and so indeed our assumption that these would eventually be satisfied in the brane-world reduction is justified. Note that the theory naturally arises with a 4-form field strength rather than the 3-form field strength that would naturally come from the $T^{3}$ reduction of the heterotic theory. This suggests that the former and the latter can be related by a strong/weak duality.

Having obtained the brane-world reduction from eight-dimensional gauged supergravity, we may now lift it back to $D=11$, by using the $S^{3}$ reduction ansatz (46). Thus we find that the eleven-dimensional fields are given in terms of seven-dimensional fields by

$$
\begin{gathered}
d \hat{s}_{11}^{2}=e^{-\sqrt{(8 / 45)} \phi} d s_{7}^{2}+e^{\sqrt{(5 / 18)} \phi}\left[W^{-(2 / 3)} d z^{2}+g^{-2} W^{4 / 3} d \Omega_{3}^{2}\right], \\
\hat{A}_{(3)}=A_{(3)}+\frac{1}{4 \sqrt{2}} g^{-2} W^{4 / 3} F_{(2)}^{i} \wedge \sigma_{i} .
\end{gathered}
$$

It is interesting to note that if we perform a coordinate transformation from $z$ to $r$, defined by $W^{-(1 / 3)} d z=d r$, and hence

$$
W=(g r)^{3 / 2}
$$

then this ansatz for the reduction from $D=11$ to $D=7$ becomes

$$
\begin{gathered}
d \hat{s}_{11}^{2}=e^{-\sqrt{8 / 45} \phi} d s_{7}^{2}+e^{\sqrt{5 / 18} \phi}\left(d r^{2}+r^{2} d \Omega_{3}^{2}\right), \\
\hat{A}_{(3)}=A_{(3)}+\frac{1}{4 \sqrt{2}} r^{2} F_{(2)}^{i} \wedge \sigma_{i} .
\end{gathered}
$$

This is recognizable as a standard type of Kaluza-Klein reduction on $T^{4}$, in which a truncation to the fields of $N=2$ supergravity in $D=7$ has been performed.

\section{B. Brane-world reduction to $D=7$, from type IIA supergravity}

Taking the results of the previous subsection, we can perform an additional $S^{1}$ Kaluza-Klein reduction on the Hopf fibers of the compactifying 3-sphere that was used in the reduction from $D=11$ to $D=8$, thereby allowing us to obtain a brane-world reduction to $D=7$ that can be viewed as coming from type IIA supergravity compactified first on $S^{2}$.

To implement this procedure, we first specialize some results for the Hopf reduction of $S^{3}$ that were obtained in Ref. 17. In terms of Euler angles $(\theta, \varphi, \psi)$, the three left-invariant 1-forms of $\mathrm{SU}(2)$ can be written as

$$
\begin{gathered}
\sigma_{1}=\cos \psi d \theta+\sin \psi \sin \theta d \varphi, \\
\sigma_{2}=-\sin \psi d \theta+\cos \psi \sin \theta d \varphi, \\
\sigma_{3}=d \psi+\cos \theta d \varphi .
\end{gathered}
$$

Clearly $\partial / \partial \varphi$ is a Killing vector for the 3-sphere metric $d \Omega_{3}^{2}=\frac{1}{4} \sigma_{i} \sigma_{i}$, and it also leaves the 3-form ansatz in (56) invariant. Let $\mu_{i}$ be three coordinates on $\mathbb{R}^{3}$ subject to the constraint $\mu_{i} \mu_{i}=1$, given in terms of $\theta$ and $\psi$ by

$$
\mu_{1}=\sin \theta \sin \psi, \quad \mu_{2}=\sin \theta \cos \psi, \quad \mu_{3}=\cos \theta .
$$


It is easily seen that in terms of these we can write the left-invariant 1-forms as

$$
\sigma_{i}=-\epsilon_{i j k} \mu_{j} d \mu_{k}+\mu_{i}(d \varphi+\cos \theta d \psi) .
$$

We can now perform a Kaluza-Klein $S^{1}$ reduction of the eleven-dimensional expressions (56) on the Hopf fiber coordinate $\varphi$, using the standard ansatz,

$$
\begin{gathered}
d \hat{s}_{11}^{2}=e^{-(1 / 6) \Phi} d \bar{s}_{10}^{2}+e^{(4 / 3) \Phi}\left(d \varphi+\overline{\mathcal{A}}_{(1)}\right)^{2}, \\
\hat{A}_{(3)}=\bar{A}_{(3)}+\bar{A}_{(2)} \wedge\left(d \varphi+\overline{\mathcal{A}}_{(1)}\right),
\end{gathered}
$$

where $\Phi$ is the type IIA dilaton. Using (61), we therefore obtain the following reduction ansatz for the fields of type IIA supergravity:

$$
\begin{gathered}
d \bar{s}_{10}^{2}=W^{(1 / 6)}\left[e^{-(9 / 8 \sqrt{10}) \phi} d s_{7}^{2}+W^{-(2 / 3)} e^{(15 / 8 \sqrt{10}) \phi} d z^{2}+\frac{1}{4} g^{-2} W^{4 / 3} e^{(15 / 8 \sqrt{10}) \phi} d \Omega_{2}^{2}\right], \\
\bar{A}_{(3)}=A_{(3)}+\frac{1}{4 \sqrt{2}} g^{-2} W^{4 / 3} \epsilon_{i j k} \mu_{i} F_{(2)}^{j} \wedge d \mu_{k}, \\
\bar{A}_{(2)}=\frac{1}{2 \sqrt{2}} g^{-1} W^{4 / 3} \mu_{i} F_{(2)}^{i}, \\
\overline{\mathcal{A}}_{(1)}=\frac{1}{2} g^{-1} \cos \theta d \psi, \\
e^{\Phi}=W e^{(5 / 4 \sqrt{10}) \phi},
\end{gathered}
$$

where the unit 2-sphere metric $d \Omega_{2}^{2}$ is given by

$$
d \Omega_{2}^{2}=d \mu_{i} d \mu_{i}=d \theta^{2}+\sin ^{2} \theta d \psi^{2} .
$$

The "vacuum" solution, corresponding to the metric (63) with $\phi=0$, can be viewed as the near-horizon limit of a D6-brane.

\section{Branes on the D6-brane}

The $D=7, N=2$ supergravity admits membrane and string solutions supported by electric or magnetic charges for $F_{(4)}$. When lifted back to $D=11$, the membrane becomes an M2-brane delocalised on a 4-hyperplane, whilst the string can be viewed as an M5-brane wrapped on the 4-hyperplane. The seven-dimensional theory also admits black hole and 3-brane solutions, which can be viewed as intersections of two M2-branes, and intersections of two M5-branes, respectively. From the type IIA point of view, they can be viewed as membranes, strings, black holes or 3-branes living in a D6-brane.

\section{REDUCTIONS OF GAUGED MAXIMAL $D=7$ SUPERGRAVITY}

\section{A. Gauged maximal seven-dimensional supergravity}

The bosonic Lagrangian for maximal $\mathrm{SO}(5)$-gauged supergravity in $D=7$ can be written as

$$
\begin{gathered}
\mathcal{L}_{7}=\hat{R} * 1-\frac{1}{4} T_{i j}^{-1} * D T_{j k} \wedge T_{k l}^{-1} D T_{l i}-\frac{1}{4} T_{i k}^{-1} T_{j l}^{-1} * \hat{F}_{(2)}^{i j} \wedge \hat{F}_{(2)}^{k l}-\frac{1}{2} T_{i j} * \hat{S}_{(3)}^{i} \wedge \hat{S}_{(3)}^{j} \\
+\frac{1}{2 g} \hat{S}_{(3)}^{i} \wedge \hat{H}_{(4)}^{i}-\frac{1}{8 g} \epsilon_{i j_{1} \cdots j_{4}} \hat{S}_{(3)}^{i} \wedge \hat{F}_{(2)}^{j_{1} j_{2}} \wedge \hat{F}_{(2)}^{j_{3} j_{4}}+\frac{1}{g} \Omega_{(7)}-V * 1
\end{gathered}
$$


where

$$
\hat{H}_{(4)}^{i} \equiv D \hat{S}_{(3)}^{i}=d \hat{S}_{(3)}^{i}+g \hat{A}_{(1)}^{i j} \wedge \hat{S}_{(3)}^{j} .
$$

The potential $V$ is given by

$$
V=\frac{1}{2} g^{2}\left(2 T_{i j} T_{i j}-\left(T_{i i}\right)^{2}\right),
$$

and $\Omega_{(7)}$ is a Chern-Simons-type of term built from the Yang-Mills fields, which has the property that its variation with respect to $\hat{A}_{(1)}^{i j}$ gives

$$
\delta \Omega_{(7)}=\frac{3}{4} \delta_{i_{1} i_{2} k l}^{j_{1} j_{2} j_{3} j_{4}} \hat{F}_{(2)}^{i_{1} i_{2}} \wedge \hat{F}_{(2)}^{j_{1} j_{2}} \wedge \hat{F}_{(2)}^{j_{3} j_{4}} \wedge \delta \hat{A}_{(1)}^{k l}
$$

An explicit expression for $\Omega_{(7)}$ can be found in Ref. 18 Note that the $S_{(3)}^{i}$ are viewed as fundamental fields in the Lagrangian. The symmetric unimodular SO(5)-valued tensor $T_{i j}$ describes the 14 scalar fields.

Let us now set the $\mathrm{SO}(5)$ Yang-Mills potentials $A_{(1)}^{i j}$ to zero, and take the scalars to be trivial also, $T_{i j}=\delta_{i j}$. This is not in general a consistent truncation, since the remaining fields $\hat{S}_{(3)}^{i}$ would act as sources for the Yang-Mills and scalar fields that have been set to zero. If we impose that these source terms vanish, i.e.,

$$
\hat{S}_{(3)}^{i} \wedge \hat{S}_{(3)}^{j}=0, \quad * \hat{S}_{(3)}^{i} \wedge \hat{S}_{(3)}^{j}=0,
$$

then the truncation will be consistent. (As we shall see below, these sources terms will indeed vanish in the brane-world reduction that we shall be considering.) The remaining equations of motion following from (65) are then

$$
\begin{gathered}
d * \hat{S}_{(3)}^{i}=0, \quad d \hat{S}_{(3)}^{i}=g * \hat{S}_{(3)}^{i}, \\
\hat{R}_{A B}=\frac{1}{4}\left(\hat{S}_{A C D}^{i} \hat{S}_{B}^{i C D}-\frac{2}{15}\left(S_{(3)}^{i}\right)^{2} \hat{g}_{A B}\right)-\frac{3}{2} g^{2} \hat{g}_{A B} .
\end{gathered}
$$

\section{B. Chiral $N=(2,0)$ supergravity from $D=7$}

We find that the following Kaluza-Klein ansatz for the seven-dimensional fields yields a consistent reduction to six dimensions,

$$
\begin{gathered}
d \hat{s}_{7}^{2}=e^{-2 k|z|} d s_{6}^{2}+d z^{2}, \\
\hat{S}_{(3)}^{i}=e^{-2 k|z|} F_{(3)}^{i}, \\
\hat{A}_{(1)}^{i j}=0, \quad T_{i j}=\delta_{i j},
\end{gathered}
$$

where the constant $k$ is related to the gauge coupling constant $g$ by

$$
g=\left\{\begin{array}{lc}
-2 k, & z>0, \\
+2 k, & z<0 .
\end{array}\right.
$$

Substituting this ansatz into the field equations of seven-dimensional $\mathrm{SO}(5)$-gauged supergravity, we find that all the equations are consistently satisfied provided that the six-dimensional fields $d s_{6}^{2}$ and $F_{(3)}^{i}$ satisfy the equations of motion of six-dimensional ungauged $N=(2,0)$ chiral supergravity, namely,

$$
F_{(3)}^{i}=* F_{3}^{i}, \quad d F_{(3)}^{i}=0, \quad R_{\mu \nu}=\frac{1}{4} F_{\mu \rho \sigma}^{i} F_{\nu}^{i \rho \sigma} .
$$


Note that the self-duality of the 3-forms ensures that the constraints (69) are indeed satisfied, since $F_{(3)}^{i} \wedge F_{(3)}^{j}=0$ for any pair of self-dual 3-forms. Of course the self-duality of the $F_{(3)}^{i}$ fields also implies one cannot write a covariant Lagrangian for this theory.

Since we know the exact embedding of seven-dimensional maximal $\mathrm{SO}(5)$-gauged supergravity in $D=11$, via the $S^{4}$ reduction, we can lift the above ansatz to an embedding in elevendimensional supergravity. Using the $S^{4}$ reduction ansatz, we therefore obtain

$$
\begin{gathered}
d \hat{s}_{11}^{2}=e^{-2 k|z|} d s_{6}^{2}+d z^{2}+g^{-2} d \mu_{i} d \mu_{i}, \\
\hat{F}_{(4)}=\frac{1}{8 g^{3}} \epsilon_{i_{1} \cdots i_{5}} \mu_{i_{1}} d \mu_{i_{2}} \wedge \cdots \wedge d \mu_{i_{5}}-g^{-1} d\left(\mu_{i} e^{-2 k|z|} F_{(3)}^{i}\right),
\end{gathered}
$$

where $\mu_{i}$ are coordinates on $\mathbb{R}^{5}$, subject to the constraint

$$
\mu_{i} \mu_{i}=1,
$$

which defines the unit 4-sphere.

\section{Five-dimensional $N=4$ ungauged supergravity from $S O(5)$-gauged $D=6$ supergravity}

By dimensionally reducing the embedding (74) of six-dimensional chiral $N=(2,0)$ supergravity on a circle in the six-dimensional space-time, we can obtain an embedding of five-dimensional $N=4$ supergravity in type IIA supergravity. Thus we begin by performing a standard $S^{1}$ KaluzaKlein reduction of the six-dimensional fields,

$$
\begin{gathered}
d s_{6}^{2}=e^{-2 \alpha \phi} d \widetilde{s}_{5}^{2}+e^{6 \alpha \phi}\left(d x_{5}+\mathcal{A}_{(1)}\right)^{2}, \\
F_{(3)}^{i}=e^{-4 \alpha \phi_{\Im} \widetilde{F}_{(2)}^{i}+\widetilde{F}_{(2)}^{i} \wedge\left(d x_{5}+\mathcal{A}_{(1)}\right),}
\end{gathered}
$$

where $\alpha=1 /(2 \sqrt{6})$ and $\widetilde{F}_{(2)}^{i}=d \widetilde{A}_{(1)}^{i}$. (Note that the form of the reduction ansatz for the sixdimensional fields $F_{(3)}^{i}$ is dictated by the fact that they are self-dual.) The theory that results from this dimensional reduction is ungauged $N=4$ supergravity in $D=5$. It is straightforward to show that the equations of motion in $D=5$ that follow from substituting (76) into (73) are derivable from the Lagrangian,

$$
\mathcal{L}_{5}=\widetilde{R} ¥ 1-\frac{1}{2} \approx d \phi \wedge d \phi-\frac{1}{2} e^{-4 \alpha \phi} \widetilde{F}_{(2)}^{i} \wedge \widetilde{F}_{(2)}^{i}-\frac{1}{2} e^{8 \alpha \phi} \widetilde{F}_{(2)} \wedge \mathcal{F}_{(2)}-\frac{1}{2} \widetilde{F}_{(2)}^{i} \wedge \widetilde{F}_{(2)}^{i} \wedge \mathcal{A}_{(1)},
$$

where $\mathcal{F}_{(2)}=d \mathcal{A}_{(1)}$.

We now substitute (76) into (74), and compare it with a standard Kaluza-Klein $S^{1}$ reduction from $D=11$ to $D=10$,

$$
\begin{gathered}
d \hat{s}_{11}^{2}=e^{-(1 / 6) \Phi} d s_{10}^{2}+e^{(4 / 3) \Phi}\left(d x_{5}+A_{(1)}\right)^{2}, \\
\hat{F}_{(4)}=F_{(4)}+F_{(3)} \wedge\left(d x_{5}+A_{(1)}\right) .
\end{gathered}
$$

By doing this, we arrive at the ansatz for the embedding of the five-dimensional ungauged $N$ $=4$ supergravity in type IIA supergravity, 


$$
\begin{gathered}
d s_{10}^{2}=e^{-(9 / 4) k|z|-(5 / 4) \alpha \phi} d \widetilde{s}_{5}^{2}+e^{-(1 / 4) k|z|+(3 / 4) \alpha \phi} d z^{2}+g^{-2} e^{-(1 / 4) k|z|+(3 / 4) \alpha \phi} d \mu_{i} d \mu_{i}, \\
F_{(4)}=\frac{1}{8 g^{3}} \epsilon_{i_{1} \cdots i_{5}} \mu_{i_{1}} d \mu_{i_{2}} \wedge \cdots \wedge d \mu_{i_{5}}-g^{-1} e^{-4 \alpha \phi} d\left(\mu_{i} e^{\left.-2 k|z| \widetilde{F}_{(2)}^{i}\right),}\right. \\
F_{(3)}=-g^{-1} d\left(\mu_{i} e^{-2 k|z|} \widetilde{F}_{(2)}^{i}\right), \\
F_{2}=\mathcal{F}_{(2)}, \quad e^{\Phi}=e^{-(3 / 4) k|z|} e^{(9 / 2) \alpha \phi} .
\end{gathered}
$$

\section{Chiral $N=(2,0)$ supergravity from type IIA NS5-brane}

We showed in Sec. V B that the chiral $(2,0)$ six-dimensional supergravity can be obtained as a brane-world Kaluza-Klein reduction from maximal gauged supergravity in $D=7$, and in turn, this can be obtained as an $S^{4}$ reduction from $D=11$. It was shown recently that one can take a singular limit of the $S^{4}$ reduction of eleven-dimensional supergravity, in which the 4-sphere degenerates to $S^{3} \times \mathbb{R} .{ }^{19}$ The reduction can then be reinterpreted as an $S^{3}$ reduction of type IIA supergravity, yielding a maximal $\mathrm{SO}(4)$-gauged supergravity in $D=7$ that admits a domain-wall, but not $A d S_{7}$, as a solution. One may refer to this theory as a "domain-wall" supergravity.

By applying this limiting procedure in the context of the brane-world reduction to the $(2,0)$ supergravity in $D=6$ that we constructed in Secs. V A and V B, we can now obtain a brane-world reduction of the $D=7$ domain-wall supergravity to the $(2,0)$ theory in $D=6$. Furthermore, we can lift this back, via its $S^{3}$ embedding, to an ansatz for type IIA supergravity. Rather than repeating the details of how the singular limit is taken here, we shall simply quote and make use of the general results already obtained in Ref. 19.

We begin by considering the brane-world reduction of the maximal seven-dimensional $\mathrm{SO}(4)$ gauged domain-wall supergravity. As in our previous examples, many of the fields are set to zero in the brane-world reduction, and so rather than presenting the full seven-dimensional theory obtained in Ref. 19, we shall instead give it in an already-truncated form, where all but the participating fields have already been set to zero. As usual, we should add the cautionary remark that one cannot in general consistently set these fields to zero while allowing the remaining fields to take generic configurations. But in anticipation of the fact that the brane-world reduction will be consistent, we can make the truncation provided that we take note also of the consequent required constraints, which will be satisfied by the brane-world reduction ansatz.

In this spirit, we therefore set to zero all the fields of the seven-dimensional $\mathrm{SO}(4)$-gauged domain-wall supergravity constructed in Ref. 19 except for the metric, the dilatonic scalar field $\phi$, and the 3 -forms $S_{(3)}^{0}$ and $S_{(3)}^{\alpha}$. Note that $S_{(3)}^{0}$ is viewed as a 3-form field strength that is derived from a 2-form potential, whilst the four 3-forms $S_{(3)}^{\alpha}$ are viewed as independent fields in their own right, which satisfy first-order equations of motion. Defining $\gamma=\sqrt{2 / 5}$, we read off from Ref. 19 that the equations of motion for these remaining fields will be

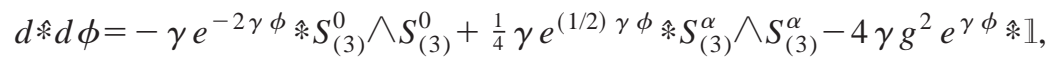

$$
\begin{aligned}
& d\left(e^{-2 \gamma \phi_{*} S_{(3)}}\right)=0, \quad d S_{(3)}^{0}=0, \\
& d S_{(3)}^{\alpha}=g e^{1 / 2 \gamma \phi_{*}} S_{(3)}^{\alpha}, \\
& \hat{R}_{M N}=\frac{1}{2} \partial_{M} \phi \partial_{N} \phi+\frac{1}{4} e^{-2 \gamma \phi}\left[S_{M P Q}^{0} S_{N}^{0 P Q}-\frac{2}{15}\left(S_{(3)}^{0}\right)^{2} \hat{g}_{M N}\right] \\
& +\frac{1}{4} e^{(1 / 2) \gamma \phi}\left[S_{M P Q}^{\alpha} S_{N}^{\alpha P Q}-\frac{2}{15}\left(S_{(3)}^{\alpha}\right)^{2} \hat{g}_{M N}\right]-\frac{4}{5} g^{2} e^{\gamma \phi} \hat{g}_{M N} .
\end{aligned}
$$

(Note that we have placed hats on all quantities associated with the seven-dimensional metric, in anticipation of the upcoming brane-world reduction to $D=6$.) 
We may first note that these equations of motion admit a domain-wall solution given by $d \hat{s}_{7}^{2}=W^{2} d x \cdot d x+d z^{2}, e^{-\gamma \phi}=W^{2}$, where $W=1+k|z|$ and $k^{2}=4 g^{2} / 25$. This provides the basis for the brane-world reduction ansatz to $D=6$. Specifically, we find that all the equations given in (80) are satisfied if we make the following ansatz:

$$
\begin{gathered}
d \hat{s}_{7}^{2}=W^{2} d s_{6}^{2}+d z^{2}, \quad e^{-\gamma \phi}=W^{2}, \\
S_{(3)}^{0}=F_{(3)}^{0}, \quad S_{(3)}^{\alpha}=W^{5 / 2} F_{(3)}^{\alpha},
\end{gathered}
$$

where the fields $d s_{7}^{2}$ and $F_{(3)}^{i}$ satisfy the equations of motion of ungauged six-dimensional $(2,0)$ supergravity, as given in (73). Note that here, the index $i$ on $F_{(3)}^{i}$ runs over the five values $i$ $=(0, \alpha)$.

We can also use the results in Ref. 19 to lift the seven-dimensional fields to those of tendimensional type IIA supergravity. For the truncated system that we are considering here, we find that the embedding is simply given by

$$
\begin{gathered}
d \bar{s}_{10}^{2}=e^{(3 / 8) \gamma \phi} d s_{7}^{2}+g^{-2} e^{-(5 / 8) \gamma \phi} d \Omega_{3}^{2}, \\
e^{\Phi}=e^{(5 / 4) \gamma \phi}, \quad \bar{A}_{(1)}=0, \\
\bar{F}_{(4)}=-e^{(1 / 2) \gamma \phi} \mu_{\alpha} S_{(3)}^{\alpha}+g^{-1} S_{(3)}^{\alpha} \wedge d \mu_{\alpha}, \\
\bar{F}_{(3)}=2 g^{-3} \Omega_{(3)}+g^{-1} S_{(3)}^{0},
\end{gathered}
$$

where $\mu_{\alpha}$ denote Cartesian coordinates on $\mathbb{R}^{4}$ subject to the constraint $\mu_{\alpha} \mu_{\alpha}=1$ that defines the unit 3-sphere with metric $d \Omega_{3}^{2}$ and volume form $\Omega_{(3)}$. The barred fields are those of type IIA supergravity, with $\Phi$ being the type IIA dilaton.

Substituting our brane-world reduction ansatz (81) into this, we obtain the following braneworld embedding of six-dimensional $(2,0)$ supergravity in type IIA supergravity:

$$
\begin{gathered}
d \bar{s}_{10}^{2}=W^{(5 / 4)}\left(d s_{6}^{2}+W^{-2} d z^{2}+g^{-2} d \Omega_{(3)}^{2}\right), \\
e^{\Phi}=W^{-(5 / 2)}, \quad \bar{A}_{(1)}=0, \\
\bar{F}_{(4)}=-W^{3 / 2} \mu_{\alpha} F_{(3)}^{\alpha}+g^{-1} W^{5 / 2} F_{(3)}^{\alpha} \wedge d \mu_{\alpha}, \\
\bar{F}_{(3)}=2 g^{-3} \Omega_{(3)}+g^{-1} F_{(3)}^{0} .
\end{gathered}
$$

\section{E. Chiral $N=(1,0)$ supergravity from heterotic 5-brane}

It was shown in Refs. 20,21, 19 that one can obtain the chiral $N=(1,0)$ theory from the $N$ $=2 \mathrm{SU}(2)$-gauged supergravity in $D=7$ that admits an $A d S_{7}$ vacuum solution, via a brane-world Kaluza-Klein reduction. There is also an $\mathrm{SU}(2)$-gauged supergravity in $D=7$ that admits a domain wall instead of $A d S_{7}$ as a vacuum solution. This theory can be obtained from the $S^{3}$ reduction of $N=1$ supergravity in $D=10$, and its domain-wall solution is therefore the $S^{3}$ reduction of the heterotic 5-brane. Clearly it can also obtained from the truncation of the $N=4 \mathrm{SO}(4)$ gauged maximal supergravity discussed in the previous subsection. It is straightforward to reduce the seven-dimensional theory or the heterotic theory in $D=10$ on the world-volume of the 5-brane and obtain the chiral $N=(1,0)$ supergravity. The reduction ansatz is identical to that of Sec. VD, but with all the fields that carry the index $\alpha$ set to zero.

Both the $(2,0)$ and $(1,0)$ theories admit a self-dual string solution in $D=6$. This solution can be lifted to $D=11$, where it becomes a self-dual string living in the world-volume of M5-brane, 
which can also be viewed as an open membrane ending on the M5-brane. ${ }^{3}$ When lifted back to $D=10$ instead, it can be viewed as a self-dual string living in the NS5-brane or the heterotic 5-brane.

\section{F. $N=(1,1)$ supergravity from $D=7$ gauged supergravity}

So far, we obtained the chiral ungauged supergravity in $D=6$ from gauged supergravity in $D=7$, which itself can be obtained from $S^{4}$ reduction of M-theory, or the $S^{3}$ reduction of the type IIA or heterotic theories. There also exist gauged supergravities in $D=7$ that give rise instead to the $N=(1,1)$ nonchiral theory in six dimensions, through a brane-world Kaluza-Klein reduction. One example is the $\mathrm{SU}(2)$-gauged supergravity that is the $S^{1}$ reduction of eight-dimensional $\mathrm{SU}(2)$-gauged supergravity, which itself can be obtained from the $S^{3}$ reduction of elevendimensional supergravity, ${ }_{14}^{4}$ as we discussed in Sec. IV. This is because the brane-world reduction of the eight-dimensional gauged supergravity gives rise to $N=2$ supergravity in $D=7$. If we perform a further $S^{1}$ reduction on a brane-world direction, the bulk gauged-supergravity in $D$ $=8$ becomes a gauged $N=4$ supergravity in $D=7$, whilst the world-volume seven-dimensional ungauged theory becomes the $N=(1,1)$ ungauged theory in $D=6$.

There should also be an $\mathrm{SO}(4)$-gauged supergravity in $D=7$ that gives rise to the $N=(1,1)$ theory in $D=6$. This can be obtained from the $S^{3}$ reduction of the type IIB theory. The bulk T-duality of the type IIA and type IIB theories then translates into a T-duality between the $N$ $=(1,1)$ and $N=(2,0)$ theories in the 5-brane world-volume.

\section{CONCLUSION}

In this paper, we have constructed several new examples of brane-world Kaluza-Klein reductions. Our focus was to construct the reductions with larger supersymmetry and in diverse dimensions that in general involve consistent Kaluza-Klein reductions with dilatonic codimension one objects, thus extending the results obtained in Ref. 3 in several ways. Specifically, we have shown that it is possible to construct consistent brane-world reductions of five-dimensional $N$ $=8 \mathrm{SO}(6)$-gauged supergravity to ungauged $N=4$ supergravity in $D=4$; of massive type IIA supergravity to ungauged $N=1$ supergravity in $D=9$; of eight-dimensional $N=2 \mathrm{SU}(2)$-gauged supergravity to ungauged $N=2$ supergravity in $D=7$; and of seven-dimensional $N=4 \mathrm{SO}(5)$ gauged supergravity to ungauged $N=2$ supergravity in $D=6$. In all these cases, just as in the original examples constructed in Ref. 3, the degree of ungauged supersymmetry in the lower dimension is one-half of the gauged one in the higher dimension, and in this paper we have focused mainly on the supergravity multiplets.

A simple calculation shows that for any brane-world (codimension one) reduction ansatz of the form

$$
d \hat{s}^{2}=e^{-2 k|z|} d s^{2}+d z^{2},
$$

the Riemann tensor $\hat{R}_{A B C D}$ of the $D$-dimensional metric $d \hat{s}^{2}$ satisfies

$$
\hat{R}_{A B C D} \hat{R}^{A B C D}=e^{4 k|z|} R_{a b c d} R^{a b c d}-4 k^{2} e^{2 k|z|} R+2 D(D-1) k^{4}
$$

in the bulk, where $R_{a b c d}$ and $R$ are the Riemann tensor and Ricci scalar of the reduced metric $d s^{2}$. This implies that any curvature of the lower-dimensional metric for which $R_{a b c d} R^{a b c d}$ or $R$ is nonzero, no matter how small, will lead to curvature singularities in the higher-dimensional metric on the Cauchy horizons at $z= \pm \infty$. These singularities were discussed in detail for a Schwarzschild black hole on the brane in Ref. 22. In Ref. 23, it was argued that such curvature singularities on the horizons arise as an artifact of considering only the zero-mode of the metric tensor, and that if the massive Kaluza-Klein modes are taken into account they could actually become dominant near the horizons, and may lead to a finite curvature there. The results of Ref. 3 and this paper suggest that the phenomenon of diverging curvature on the Cauchy horizons for the AdS domain wall reductions (or null horizons for the dilatonic domain wall reductions) may be more severe. 
Specifically these results show that the brane-world reductions correspond to exact fully nonlinear consistent embeddings in which the massive Kaluza-Klein modes can be consistently decoupled. This implies that there certainly exist exact solutions on the brane-world where Kaluza-Klein modes do not enter the picture, even at the nonlinear level. For these solutions, the curvature will inevitably diverge at the horizons. It becomes necessary, therefore, either to live with these (null) singularities or else to find a principle, perhaps based on the imposition of appropriate boundary conditions, for rejecting the solutions of this type. (Let us also remark that a deviation of the dilatonic domain wall solutions from the flat (BPS)-limit generically introduces naked singularities, ${ }^{24}$ again pointing towards difficulties with the interpretation of such solutions within a more realistic set-up.) It should be emphasized, however, that regardless of the physical questions that are prompted by these results, the brane-world Kaluza-Klein reductions remain valid mathematical constructs in their own right. In fact as relatively simple examples of consistent reductions that have no obvious group-theoretic explanation, they can be viewed as precursors of the remarkable examples of consistent reductions on spheres.

Finally, we again emphasize that the absolute-value sign in Eq. (84) for the brane-world metrics in the AdS codimension one brane (as well as dilatonic examples as discussed in the text) actually requires an explicit delta function source to support such a $Z_{2}$-symmetric codimension one object that in turn allows for the trapping of gravity on the world-volume of the brane (at $z$ $=0$ ). The understanding of such delta-function sources in the lower dimension may require a subtle interpretation in terms of fundamental sources, such as D-brane sources of the higher dimensional theory. ${ }^{25}$ Nevertheless, the consistency of the Kaluza-Klein reduction in the bulk (for $z \neq 0)$ is valid quite independently of the origin of the domain wall sources.

\section{ACKNOWLEDGMENTS}

We would like to thank Gary Gibbons, Chris Hull, Jim Liu, and Kelly Stelle for discussions. M.C. was supported in part by DOE Grant No. DE-FG02-95ER40893 and NATO Grant No. 976951. H.L. was supported in full by DOE Grant No. DE-FG02-95ER40899. C.N.P. was supported in part by DOE Grant No. DE-FG03-95ER40917.

${ }^{1}$ L. Randall and R. Sundrum, “An alternative to compactification,” Phys. Rev. Lett. 83, 4690 (1999), hep-th/9906064.

${ }^{2}$ M. Cvetič and H.H. Soleng, "Supergravity domain walls," Phys. Rep. 282, 159 (1997), hep-th/9604090.

${ }^{3}$ H. Lü and C.N. Pope, "Branes on the brane," hep-th/0008050.

${ }^{4}$ D. Youm, "Solitons in brane worlds," Nucl. Phys. B 576, 106 (2000), hep-th/9911218.

${ }^{5}$ M. Cvetič, H. Lü, and C.N. Pope, "Domain walls with localized gravity and domain-wall/QFT correspondence," hep-th/0007209.

${ }^{6}$ A. Lukas, B.A. Ovrut, K.S. Stelle, and D. Waldram, “'The universe as a domain wall,” Phys. Rev. D 59, 86001 (1999), hep-th/9803235.

${ }^{7}$ P. Hořava and E. Witten, “Heterotic and type I string dynamics from eleven-dimensions,” Nucl. Phys. B 460, 506 (1996), hep-th/9510209.

${ }^{8}$ R. Kallosh, A. Linde, T. Ortin, A. Peet, and A. Van Proeyen, “Supersymmetry as a cosmic censor,” Phys. Rev. D 46, 5278 (1992), hep-th/9205027.

${ }^{9}$ M. Cvetič and D. Youm, "Dyonic BPS saturated black holes of heterotic string on a six torus," Phys. Rev. D 53, 584 (1996), hep-th/9507090.

${ }^{10}$ L.J. Romans, “Massive $N=2 a$ supergravity in ten dimensions,'” Phys. Lett. B 169, 374 (1986).

${ }^{11}$ Bergshoeff, M. de Roo, M.B. Green, G. Papadopoulos, and P.K. Townsend, "Duality of type II 7-brane and 8-branes," Nucl. Phys. B 470, 113 (1996), hep-th/9601150.

${ }^{12}$ I.V. Lavrinenko, H. Lü, C.N. Pope, and K.S. Stelle, "Superdualities, brane tensions and massive type IIA/IIB duality," Nucl. Phys. B 555, 201 (1999).

${ }^{13}$ G. Papadopoulos, J.G. Russo, and A.A. Tseytlin, “Curved branes from string dualities,” Class. Quantum Grav. 17, 1713 (2000), hep-th/9911253.

${ }^{14}$ A. Salam and E. Sezgin, “ $d=8$ supergravity,” Nucl. Phys. B 258, 284 (1985).

${ }^{15}$ J. Schwerk and J.H. Schwarz, "How to get masses from extra dimensions," Nucl. Phys. B 153, 61 (1979).

${ }^{16}$ M. Cvetič, H. Lü, and C.N. Pope, "Consistent sphere Kaluza-Klein reductions," Phys. Rev. D 62, 64028 (2000), hep-th/0003286

${ }^{17}$ M. Cvetič, H. Lü, and C.N. Pope, “Consistent warped Kaluza-Klein reductions, half-maximal gauged supergravities, and $C P^{n}$ constructions," hep-th/0007109.

${ }^{18}$ M. Pernici, K. Pilch, and P. van Nieuwenhuizen, "Gauged maximally extended supergravity in seven dimensions," Phys. Lett. B 143, 103 (1984). 
${ }^{19}$ M. Cvetič, H. Lü, C.N. Pope, A. Sadrzadeh, and T.A. Tran, " $S^{3}$ and $S^{4}$ reductions of type IIA supergravity," hep-th/0005137.

${ }^{20}$ M. Cvetič, J.T. Liu, H. Lü, and C.N. Pope, "Domain wall supergravities from sphere reduction,” Nucl. Phys. B 560, 230 (1999), hep-th/9905096.

${ }^{21}$ A.H. Chamseddine and W.A. Sabra, “ $D=7 \mathrm{SU}(2)$ gauged supergravity from $D=10$ supergravity," Phys. Lett. B 476 , 415 (2000), hep-th/9911180.

${ }^{22}$ A. Chamblin, S.W. Hawking, and H.S. Reall, "Brane world black holes," Phys. Rev. D 61, 65007 (2000), hep-th/9909205.

${ }^{23}$ S.B. Giddings, E. Katz, and L. Randall, "Linearized gravity in brane backgrounds," J. High Energy Phys. 3, 23 (2000), hep-th/0002091.

${ }^{24}$ M. Cvetič and H.H. Soleng, "Naked singularities in dilatonic domain wall space times," Phys. Rev. D 51, 5768 (1995), hep-th/9411170.

${ }^{25}$ M. Cvetič, M.J. Duff, J.T. Liu, H. Lü, C.N. Pope, and K.S. Stelle, "Randall-Sundrum branes tensions," hep-th/ 0011167. 